\title{
Les mutations dynamiques, résultat de l'expansion de séquences répétitives
}

Les mutations, et en particulier celles qui sont pathogènes, ont longtemps été considérées comme des changements affectant spécifiquement un gène, d'abord dans la partie codante, débordant ensuite dans les zones régulatrices. Une telle mutation, une fois apparue, était fixe et statique, transmise inchangée aux générations suivantes. Ce concept est bouleversé par l'irruption d'une nouvelle classe de mutations : le changement du nombre de répétitions courtes, à proximité ou à l'intérieur d'un gène, qui semble avoir pour effet de réduire ou même de supprimer l'expression de ce gène. Les lecteurs de $\mathrm{m} / \mathrm{s}$ connaissent l'essor des mini- et microsatellites, répétitions en tandem de courtes séquences en nombre variable, qui forment la base de la méthode d'individualisation appelée empreinte génétique. Jusqu'à une date récente, on ne croyait pas ces variations quantitatives susceptibles d'influencer notablement le phénotype. Mais, depuis deux ans, cette variabilité a acquis droit de cité en pathologie génétique.

Le caractère essentiel de ces variations est que la mutabilité du produit d'un changement en nombre de copies diffère de celle de son prédécesseur : audessous d'un nombre critique de répétitions, ce nombre ne varie pas dans les générations successives. Au-dessus de ce seuil apparaît une instabilité qui s'accroît au fur et à mesure que le nombre lui-même s'élève. Pour cette raison, Richards et Sutherland (Adélaïde, Australie) ont appelé ce mécanisme mutations dynamiques [1]. Il s'applique à deux, et peut-être à trois maladies génétiques dont $\mathrm{m} / \mathrm{s}$ a parlé récemment : l'X fragile $\left(n^{\circ} 4\right.$, vol. 7 , p. $379 ; n^{\circ} 3$, vol. 8, p. $252 ; n^{\circ} 9$, vol. 8, p. 878), la dystrophie myotonique de Steinert ( ${ }^{\circ} 3$, vol. 8, p. 249), l'atrophie musculaire spino-bulbaire ou maladie de Kennedy ( $0^{\circ} 7$, vol. 7, p. 752).

Le gène FMR1 (fragile $X$ mental retarda$\mathrm{m} / \mathrm{s} n^{\circ} 9 \mathrm{vol} .8$, novembre 92 tion 1) contient normalement, en amont de sa partie codante, un nombre de répétitions du triplet CCG allant de 6 à 60. Les "porteurs sains " de l'X fragile en contiennent de 60 à 200, ainsi que les femmes vectrices; les hommes affectés en ont plus de 200. En règle générale, le nombre de copies n'augmente pas d'un homme "porteur " à sa fille vectrice. C'est lorsque cette vectrice donne naissance à un garçon que se produit le phénomène d'amplification et que se révèle cliniquement la maladie.

Les recherches génétiques récentes [1, 2] ont mis en lumière le fait que, chaque fois que l'ADN des parents est disponible, on peut montrer cette transmission d'un nombre anormal de triplets CCG chez l'un d'entre eux. L'augmentation progressive du nombre de triplets avec les générations explique enfin le phénomène d'" anticipation " qui, dans le cas de l'X fragile, consiste en une pénétrance croissante dans un même pedigree. L'instabilité est en principe d'origine méiotique ; elle peut être observée à partir de 54 copies chez le parent ; il peut s'y ajouter une instabilité mitotique, au-dessus de 200 copies ; il s'ensuit l'apparition de bandes multiples chez un même individu, conduisant à la formation de trainées à l'électrophorèse; le même aspect pourra s'observer dans la dystrophie myotonique. Dans cette maladie, c'est un triplet CTG, situé dans la partie 3' non traduite du gène, sur le chromosome 19 en 19q13.2, qui est amplifié [3-6]. Là encore, le nombre normal de répétitions varie, de 5 à 27. Les signes cliniques apparaissent au-delà de 50 copies. On observe aussi l'anticipation : parallèlement à l'augmentation du nombre des répétitions, les symptômes s'aggravent et la date de leur apparition devient plus précoce. Dans cette maladie autosomique dominante, l'augmentation peut se produire, que le vecteur soit masculin ou féminin, mais elle est en général plus forte quand la maladie est transmise par une femme. On sait que la forme néonatale, d'une extrême gravité, n'apparaît que si c'est la mère qui transmet, mais on ne possède pas encore l'explication moléculaire de cette hypotonie du nouveau-né.

La situation est quelque peu différente dans l'atrophie musculaire spinobulbaire. Elle provient d'une lésion particulière du gène du récepteur des androgènes, situé sur l'X dans la partie proximale du bras long $[7,8]$. Celui-ci porte, dans sa partie codante, une répétition du triplet CAG (codant pour des glutamines), normalement au nombre de 17 à 26. Il est, chez les malades, de 40 à 52, les valeurs intermédiaires étant exceptionnelles. Les augmentations avec les générations sont plus discrètes, et peuvent manquer. Cliniquement, on observe une variabilité extrême, même à l'intérieur d'une famille, et pas de signes nets d'anticipation. La figure 1 résume les caractères principaux des effets de l'amplification dans les trois maladies.

Il serait de grande importance de pouvoir évaluer l'impact de la notion nouvelle de mutations dynamiques en pathologie. Deux facteurs peuvent jouer en sens inverse: d'une part, quelle est la fréquence des néomutations dans les maladies déjà identifiées? d'autre part, peut-on prévoir de nouvelles découvertes dans ce domaine?

1. A la première question il semble exister une réponse ferme: dans la dystrophie myotonique, on n'a jamais observé de néomutations ; de plus, plusieurs polymorphismes de restriction ont montré un fort déséquilibre de liaison avec la dystrophie myotonique; l'ensemble fait conclure à la probabilité d'une origine unique de la maladie, d'un effet fondateur [3]. A la lumière de ces observations, on a reconsidéré la notion qui attribuait à l'X fragile un taux très élevé de mutations nouvelles. Richards et al. [2] ont 


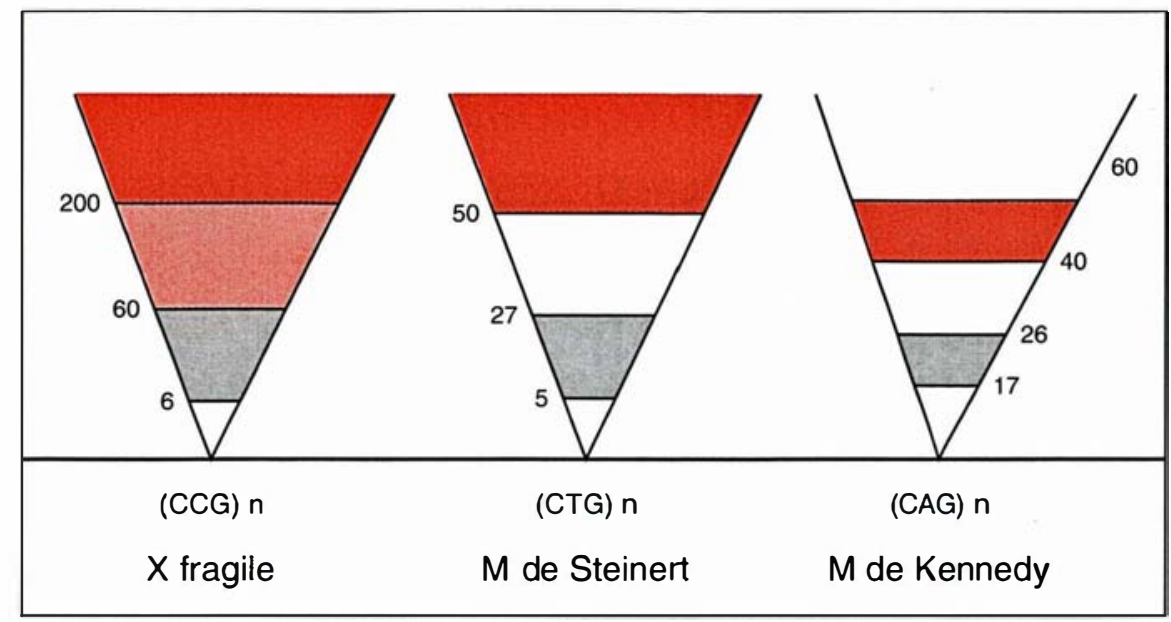

Figure 1. Nombre de répétitions de trinucléotides chez les sujets normaux, les " porteurs" et les malades. En gris : normaux. En rose : "porteurs " (seulement de l'X fragile). En rouge: malades. (Modifié d'après [1]).

comparé des haplotypes comportant des polymorphismes de la région de l'X fragile dans des cohortes de sujets avec X fragile d'Australie et des ÉtatsUnis avec des témoins normaux provenant du CEPH et des Etats-Unis. Ils ont trouvé des déséquilibres importants qui leur font conclure à un petit nombre de "fondateurs ", peut-être trois, et à la rareté de l'apparition de mutations nouvelles.

Dans le cas de la maladie de Kennedy, les données sont beaucoup moins nettes ; l'anticipation n'est pas démontrée, et les familles examinées n'ont

\begin{tabular}{|c|c|}
\hline \multicolumn{2}{|c|}{$\begin{array}{c}\text { Tableau I } \\
\text { RÉPÉTITIONS NUCLÉOTIDIQUES } \\
\text { (D'après [10]) }\end{array}$} \\
\hline Composition & $\begin{array}{l}\text { Maladies } \\
\text { génétiques }\end{array}$ \\
\hline $\begin{array}{c}\text { (AAC)n/(GTT)n } \\
\text { (AAG)n/(CTT)n } \\
\text { (AAT)n/(ATT)n } \\
\text { (ACC)n/(GGT)n } \\
\text { (ACG)n/(CGT)n } \\
\text { (ACT)n/(AGT)n } \\
\text { *AGC)n/(GCT)n } \\
\text { (AGG)n/(CCT)n } \\
\text { (ATC)n/(GAT)n } \\
\text { (CCG)n/(CGG)n }\end{array}$ & Kennedy, Steinert \\
\hline
\end{tabular}

* Les triplets écrits ici peuvent également s'écrire, par simple permutation, CAG lamplifié dans la maladie de Kennedy) et CTG (amplifié dans la myotonie de Steinert). probablement pas encore été comparées quant à leurs haplotypes. tion du gène reste encore à préciser. On a invoqué, dans l'X fragile, le rôle de la méthylation dans le triplet CCG/CGG, et il a été en effet démontré que l'absence d'expression de ce gène chez les malades peut être reliée à l'hypermutation du triplet (CCG)n hyper-répété.

Chez un fotus mâle atteint, l'amplification était observée aussi bien au niveau des villosités choriales que dans les tissus fœtaux étudiés après une interruption volontaire de grossesse Dans le tissu placentaire, cependant, ces triplets n'étaient pas méthylés, et le gène FMR1 était transcrit. Dans les tissus fotaux, en revanche, les triplets étaient méthylés, et le gène n'était pas exprimé [9]. Un tel mécanisme, cependant, ne peut être invoqué dans la maladie de Steinert car le triplet en cause n'est pas méthylable.

2. Il existe en tout dix motifs trinucléotidiques possibles de répétition, les autres s'en déduisant par complémentarité, comme le montre le Tableau I emprunté à Richards et Sutherland [10]. On peut voir que les maladies de Steinert et Kennedy mettent en jeu le même triplet AGC, bien que le transcrit soit CTG dans un cas, CAG dans l'autre. On voit également que huit triplets n'ont encore jamais été mis en cause en pathologie. Pour évaluer l'ampleur des possibilités, on peut noter que, dans une autre publication
Le mécanisme qui provoque l'inactiva-
[1], les mêmes auteurs ont dénombré 30 séquences comportant au moins cinq copies de l'un des trinucléotides, à partir de la banque de données GenBank. Ils ont aussi dressé une liste de dix exemples de répétitions du triplet CCG-CGG (en cause dans l'X fragile) dans des gènes humains, dont trois dans des régions codantes.

Une dernière question peut être soulevée : l'existence d'une séquence répétée capable d'amplification suffit-elle à déclencher le processus pathologique? Peut-être doit-elle être flanquée d'une autre séquence, capable de lui conférer cette instabilité, et dont la nature resterait à déterminer.

Jean-Claude Dreyfus

ICGM, Inserm U. 129, CHU Cochin, 24, nue du Faubourg Saint-Jacques, 75014 Paris, France.

\section{RÉFÉRENCES}

1. Richards RI, Sutherland GR. Dynamic mutations : a new class of mutations causing human disease. Cell 1992 ; 70 : 709-12.

2. Richards RI, Holman K, Friend K, et al. Evidence of founder chromosomes in fragile $\mathrm{X}$ syndrome. Nature Genet $1992 ; 1$ : 257-60.

3. Harley HG, Brook JD, Rundle SA, et al. Expansion of an unstable DNA region and phenotypic variation in myotonic dystrophy. Nature 1992 ; 355 : 545-6.

4. Brook JD, McCurrach ME, Harley HG, et al. Molecular basis of myotonic dystrophy : expansion of a trinucleotide (CTG) repeat at the 3' end of a transcript encoding a protein kinase family member. Cell 1992; 68 : 799-808.

5. Fu YH, Pizzuti A, Fenwick RG, et al. An unstable triplet repeat in a gene related to myotonic muscular dystrophy. Science 1992 ; 255: 1256-8.

6. Mahadevan M, Tsilfidis C, Sabourin L, et al. Myotonic dystrophy mutation : an unstable CTG repeat in the 3' untranslated region of the gene. Science $1992 ; 255: 1253-5$. 7. La Spada AR, Wilson EM, Lubahn DH, Harding AE, Fischbeck H. Androgen receptor gene mutations in X-linked spinal and bulbar muscular atrophy. Nature 1991; 352 : 77-9.

8. Biancalana V, Serville F, Pommier J, Julien J, Hanauer A, Mandel JL. Moderate instability of the trinucleotide repeat in spinobulbar muscular atrophy. Hum Mol Genet $1992 ; 1: 255-8$

9. Sutcliffe JS, Nelson DL, Zhang F, et al. DNA methylation represses $F M R-1$ transcription in fragile X syndrome. Hum Mol Genet 1992 ; 1 : 397-400

10. Richards RI, Sutherland GR. Heritable unstable DNA sequences. Nature Genet 1992 ; $1: 7-9$. 